\title{
Artículos
}

\section{El inventario de recursos de información como herramienta de la auditoría de información}

\author{
Por Jesús Tramullas
}
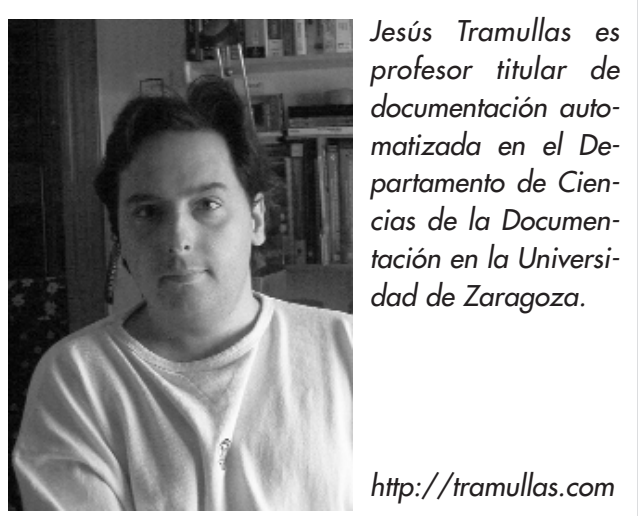

Resumen: Este trabajo desarrolla la función del inventario de recursos de información como herramienta dentro del proceso genérico de auditoría de información. Examina las propuestas de inventario presentes en la bibliografía especializada, así como el nivel de detalle de las mismas. Como conclusión propone un modelo relacional que atienda a los diferentes tipos de objetos que deben ser objeto de representación.

Palabras clave: Auditoría de información, Inventario de recursos de información, Inventario de conocimiento.

Title: Information resources inventories as a tool for information auditing

Abstract: This paper discusses the function of the information resources inventory as a tool within the process of information audits and examines the proposals for inventories that appear in the professional literature, focusing on the level of detail provided in each case. Finally, the author proposes a relational model which to address the different types of objects that may be represented.

Keywords: Information and knowledge audits, Information resources inventory.

Tramullas, Jesús. “El inventario de recursos de información como herramienta de la auditoría de información”. En: El profesional de la información, 2003, julio-agosto, v. 12, n. 4, pp. 256-260.

\section{Alcance de la auditoría de información}

La auditoría de información es una de las principales actividades a desarrollar en el marco más amplio de las iniciativas y proyectos de gestión de la información y del conocimiento (Bukowitz; Williams, 2000; Tiwana, 2002). El contexto general de esta clase de proyectos permite establecer un conjunto de fases y actividades necesarias para intentar asegurar la calidad y el éxito de la iniciativa, que pueden variar en número y denominación entre los diferentes autores que se han ocupado de la cuestión. Sin embargo, en todos ellos aparece la auditoría como una de las primeras actividades a llevar a cabo, la cual, dependiendo del contexto, es llamada tanto auditoría de la información como del conocimiento. Para el objetivo de este trabajo, y dado el contexto en el que se encuentra, se ha optado por diferenciar entre ambas, entendiendo que la primera tiene como objeto de trabajo los recursos de información explícita. Por su parte, la del conocimiento sería una ampliación de la misma, que incluiría también las fuentes de información tácita, íntimamente re- lacionados con el denominado capital intelectual, en el sentido en que esta diferencia entre tácito y explícito ha sido adoptada, con gran éxito, desde la propuesta del trabajo clásico de Nonaka y Takeuchi (1995). Esta distinción no excluye la consideración y estudio de los usuarios en el marco del proceso general.

«A systematic examination of
information use, resources and
flows, with a verification by re-
ference to both people and
existing documents, in order to
establish the extent to which
they are contributing to an or-
ganization's objectives» (Aslib)

La importancia de auditar los recursos y flujos de información no debe hacer olvidar la importancia de la información tácita o intangible. Cada vez en mayor medida se está incidiendo en la importancia de la parte humana en las auditorías de información y de cono- 
cimiento en cuanto verdaderos creadores de conocimiento en cualquier organización. Sin embargo, el enfoque adoptado en este trabajo se centra en la información explícita, puesto que se entiende que sin un profundo conocimiento de la misma, difícilmente se pueden abordar proyectos y programas de gestión del conocimiento con garantías de éxito.

Diferentes definiciones y objetivos de la auditoría de información han sido establecidos por Burck y Horton (1988), St. Clair (1997), Robertson (1997), Buchanan y Gibb (1998), Orna (1999), DiMattia y Blumenstein (2000) y Henczel (2001). Independientemente de las variaciones de enfoque o de detalle de las diferentes propuestas, cabe señalar que todas ellas coinciden en la necesidad de llevar a cabo un estudio minucioso tanto de la utilización de la información explícita disponible como de las necesidades de información detectadas, y ambas en el marco de la actividad que desarrolla una organización dada, así como de los objetivos de la misma. La auditoría de la información atiende, entonces, a recursos de información, recursos humanos (tanto productores como usuarios), flujos de información, y formulación de políticas en función de objetivos. Henczel (2000) ha establecido la relación entre estudios de usuario, auditoría de la información y auditoría del conocimiento en un proyecto de gestión del conocimiento. El alcance del estudio de auditoría que se lleve a cabo dependerá de los objetivos fijados por la organización para el mismo, entendiendo que la auditoría puede resultar global, si analiza las actividades de la entidad en su totalidad, o parcial, si atiende a un proceso de negocio específico o a un proyecto particular. Resulta evidente que este análisis entronca directamente con la extensa tradición de estudios de usuarios presente en la actividad científica de la ciencia de la información y la documentación.

\section{Perspectivas de aplicación}

La bibliografía publicada sobre auditoría de información desde 1980, aunque no es abundante, muestra la utilización de la misma en diferentes tipos de activi-

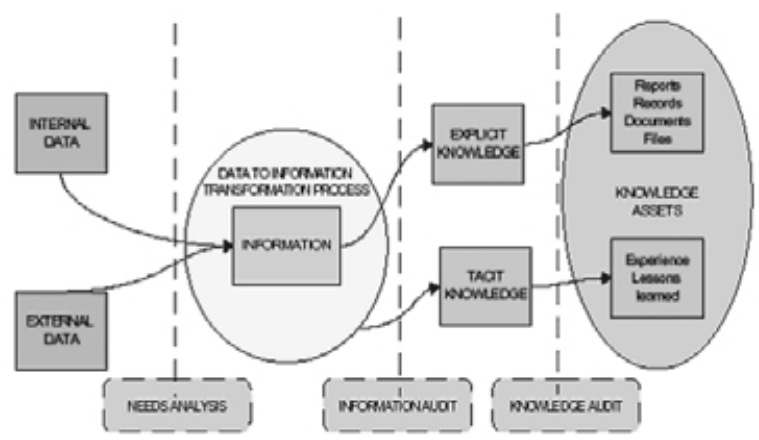

Figura 1. Del análisis de necesidades a la auditoría del conocimiento (Henczel, 2000) dades. Si en la década de 1980 los trabajos publicados insistían en su uso en el contexto de unidades de información (en especial bibliotecas y centros de documentación de empresas y organizaciones), en la década de 1990 destacan los estudios relacionados con gestión de información en las organizaciones y gestión del conocimiento. En los últimos años, el impacto y desarrollo de las intranets, los sistemas de gestión de contenidos en entornos digitales, y el auge de las comunidades de práctica, se ha visto reflejado en la aparición de técnicas y trabajos sobre la aplicación de la auditoría de la información. El diseño de productos y entornos de información digital, herramienta básica en el momento actual para cualquier empresa o administración, debe beneficiarse de la realización de auditorías de información. Por último, la importancia cada vez mayor de las actividades de I+D+I (investigación científica, desarrollo e innovación tecnológica) en las empresas y los centros de investigación, con el uso exhaustivo de recursos de información, y la vigilancia tecnológica, son otros motores del desarrollo de la auditoría de la información.

\section{«There is as yet no standard for how to do information audits»}

(Orna, 1999)

Los autores referenciados en apartados anteriores, e incluidos en la bibliografía presentada al final de este trabajo, establecen que el desarrollo de una auditoría de la información abarca diferentes actividades necesarias para asegurar la calidad del resultado final. Sin embargo, la mayor parte de los trabajos publicados establecen marcos generales de actividad, que deben ser objeto de un desarrollo posterior. Henczel (2001) propone siete fases para la auditoría de la información, correspondientes a planificación, recopilación de datos, análisis de datos, evaluación de datos, comunicación de recomendaciones, implantación de recomendaciones y revisión continua, señalando además que este esquema puede modificarse para atender a las características del entorno de aplicación. Orna (1999) establece cuatro etapas identificadas como investigación inicial, auditoría de información, hoja de balance y desarrollo de políticas. Buchanan y Gibb (1997) proponen un modelo de integración en cinco pasos identificados respectivamente con promover, identificar, analizar, contabilizar y sintetizar. Burk y Horton (1988) también proponen en su metodología InfoMap cuatro fases estándares, correspondientes a exploración, coste/valor, análisis y síntesis.

Independientemente de las variaciones de enfoque o de organización de las actividades que deben llevarse a cabo, las propuestas delineadas en el párrafo anterior ofrecen unos elementos comunes que deben ser 
destacados. En primer lugar, la auditoría de la información debe llevarse a cabo en el marco de una iniciativa de la organización, relacionada con los procesos estratégicos de la misma, con un compromiso firme de estudio y mejora. A su vez, debe incluirse en una política general de gestión de la información y/o del conocimiento, a escala corporativa. En segundo lugar, el núcleo de la auditoría de información se fundamenta en la obtención, tratamiento y análisis de datos sobre recursos de información, su utilización y su impacto en las actividades de la organización. Por último, los resultados del punto anterior se plasman en forma de memoria, informe o recomendación, con diferentes niveles de detalle, que delinea el estado actual de la gestión de información y las acciones necesarias para su optimización.

\section{«Information leverage points: information that, once identi- fied, provides a superior map of the roads to business suc- cess» (Davenport, 1996)}

El desarrollo de la auditoría y la recopilación del inventario de información hacen necesario aplicar un conjunto de técnicas de toma o captura de datos. La revisión de la bibliografía sobre auditoría de la información permite constatar que en principio nos centramos en aquella explicitada en recursos y soportes cuantificables. En contraposición, las técnicas que se utilizan para estudiarla descansan esencialmente sobre actividades humanas. Con mayor o menor detalle, los diferentes autores citados en este trabajo proponen técnicas de descubrimiento y descripción basadas en formularios, entrevistas, grupos de enfoque (focus groups), observación de actividades, etc., que pueden ser llevadas a cabo por grupos de trabajo internos y/o externos. A ellas hay que añadir, cada vez en mayor medida, el análisis cuantitativo y cualitativo de la actividad registrada de uso de herramientas y recursos de información como por ejemplo los ficheros de log de una intranet, que reflejan los comportamientos reales de los usuarios.

\section{Productos de la auditoría de información}

La auditoría de información ofrece como resultado 3 tipos de productos que pueden identificarse como inventario de información, mapa de información y memoria o informe final. La memoria o informe es la síntesis final del proceso de auditoría y contiene un estado de los recursos de información, de su utilización, de los flujos de información detectados y de las recomendaciones necesarias para llevar a cabo una gestión estratégica de la información en el marco de la organización.

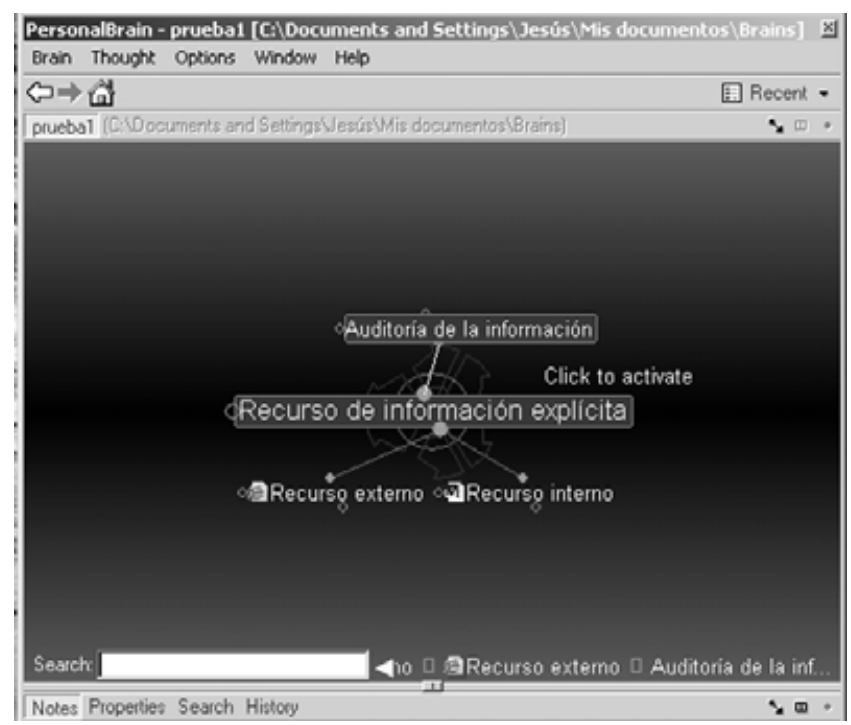

Figura 2. Combinación de inventario y mapa en un entorno gráfico

En numerosas ocasiones es identificada con el mapa de información, que en principio es una representación gráfica, bidimensional o tridimensional, de los procesos de actividad desarrollados, con indicación expresa de las personas o grupos de personas que participan en los mismos, así como la utilización que hacen de los recursos de información explícita disponibles. Otros desarrollos de esta técnica que inciden más en los aspectos de recursos humanos y en la información tácita reciben el nombre de mapas de conocimiento, aunque en gran número de ocasiones ambas expresiones suelen usarse como sinónimas. Sin embargo, un mapa de información o de conocimiento muestra una imagen del estado real, en un momento dado, de los procesos y flujos de información. Es necesario completar el mismo con las recomendaciones pertinentes para mejorar la utilización de los recursos y de los flujos de información, y estas recomendaciones se incluyen en la memoria o informe de auditoría.

Por otra parte, diferentes autores unen el inventario de información con el mapa de información, entendiendo que ambos son inseparables y existen los medios tecnológicos que permiten su desarrollo conjunto (Stevens, 2000; Skyrme, 2002). Esta diferente concepción y uso de los términos también ha sido señalada por Dobson (2002), pudiendo apreciarse cuando se realiza un búsqueda de los términos en las principales fuentes de información disponibles sobre la gestión de información y la gestión del conocimiento.

\section{Inventario de recursos de información}

Es la herramienta fundamental para la auditoría de información. Los procesos de toma de datos alimentan el inventario; los procesos de análisis consideran los datos incluidos en el mismo. El nivel de detalle de los elementos contenidos en el inventario influirá determinantemente en el resultado de la auditoría, tanto en lo 
referido al mapa de información, como en lo concerniente a la memoria final.

A pesar de esta reconocida importancia, lo cierto es que el inventario no ha recibido, en la bibliografía sobre la cuestión, la atención que sería deseable. Burk y Horton (1988) ofrecen varios modelos de plantillas de descripción de recursos y procesos de información, configurando lo que denominaron IRE (information resource entity), sobre las que posteriormente desarrollaron un sistema de bases de datos mediante $d$ Base $I V$, gracias a una aplicación informática a la que llamaron Infomapper. Sin embargo, estos autores insistían en que se creaba, en un primer momento, un "inventario preliminar" y que, tras varias fases de descubrimiento de información, los datos del mismo eran completados hasta un nivel de detalle que establecía el propio desarrollo del proceso de auditoría.

Sin embargo, el nivel de este desarrollo posterior no aparece claramente explicitado en el texto. Buchanan y Gibb (1997) han propuesto un modelo de proceso más detallado que podría implementarse sobre un sistema de base de datos. Para Henczel (2001), el inventario, al que llama information resources database, se crearía y completaría dentro de su fase segunda. Para ello, sigue la propuesta de estructura de bases de datos para recursos de información realizada por Buchanan (1999) a la que añade la necesidad de usar un sistema relacional de gestión de bases de datos, con la finalidad de crear relaciones entre registros de recursos de información, tareas, objetivos, factores críticos de éxito y unidades de negocio.

Aunque no ofrece esquemas para estos tipos de registros, resulta evidente que el inventario debe incorporar en su representación el contexto de los recursos de información. Cabe destacar que el texto de esta autora incorpora un estudio de caso precisamente sobre el desarrollo y la interpretación de un inventario de información, aunque sin abordar el nivel de detalle de la estructura. En esta necesidad de completar el inventario de recursos de información incide la consultora David Skyrme Associates (2002), una de las más prestigiosas en el campo de la gestión del conocimiento, al indicar la incorporación en el inventario de conocimiento (knowledge inventory) de flujos y usos, personas responsables, usuarios, etc., para obtener la necesaria visión global. Orna (1999) no establece ningún esquema para el inventario de información o conocimiento, pero insiste en la necesidad de disponer previamente de una clara estructura formal de registro para los datos obtenidos durante la fase de recopilación con la finalidad de facilitar su procesamiento y análisis posterior.

Para Applehans, Globe y Laugero (1999), el objetivo de la auditoría sería localizar y documentar los llamados information leverage points, (ILPs) (noción que toman de la propuesta de Davenport en $\mathrm{CIO} \mathrm{ma}$ gazine, 1996, junio). Estos autores, aunque no abordan directamente la cuestión del inventario de recursos de información, deben tenerse en consideración ya que la estructura del método que proponen facilita la creación de un soporte de bases de datos para la obtención del inventario. Los objetos de registro detallado a contemplar serán los procesos, los usuarios de información, los centros de contenido, los satélites de contenido y los propietarios del contenido. De esta forma se completaría el inventario de información con la introducción de los usuarios, en la línea sugerida por Skyrme en el párrafo anterior. En el mismo sentido se encuentra la iniciativa de Hylton (2002) sobre auditoría del conocimiento, quien avanza un paso más al incluir en la misma el estudio no sólo de los recursos de información que se utilizan, sino también los propios procesos internos de captura y creación que tienen lugar en la organización.

\section{«This stage of the knowledge audit fully incoporates the core science of knowledge auditing, which involves a knowledge in- ventory, building a knowledge map, charting the flow of know- ledge and conducting knowled- ge gap analysis» (Hylton, 2002)}

Una propuesta más exhaustiva para la elaboración de un inventario de información, o de recursos de información, es la llevada a cabo por la administración australiana, a través del Office of Information Technology, Department of Information Technology and Management (1997-2002). Tras determinar los objetivos del inventario, establece que debe identificar objetos de información a dos niveles, denominados conceptual y físico respectivamente. El primero describe las necesidades de información, mientras que el físico describe la información disponible en el estado actual. En consecuencia, presenta dos inventarios: uno para el nivel conceptual, que sería el modelo de información requerido, y otro para el físico, correspondiente a la descripción de las fuentes de información disponibles en el estado actual. En este caso, el directorio de información actúa como base de datos de referencia ya que contendrá una descripción detallada de los recursos disponibles, organizados mediante una clasificación, tesauro o taxonomía propia de la organización que se trate. Una vez disponibles ambos inventarios, se analizarán los information gaps (lagunas o interrupciones de información) que se detecten en la comparación entre ambos. 
Sin embargo, y de cara a la identificación, clasificación y descripción de los recursos de información, es poca la bibliografía disponible, aunque resulta evidente que esta cuestión es clave para plantear la creación y desarrollo de un inventario de información o de conocimiento. Recientemente, Holsapple y Joshi (2001) han abordado los diferentes tipos de knowledge assets que pueden encontrarse en una organización. Estos autores han llevado a cabo un estudio Delphi para identificar los tipos de recursos existentes y de la clasificación establecida interesan, desde nuestra perspectiva, los llamados recursos de contenido que se dividen en participantes y "artefactos". Entre los participantes hay que destacar que la propuesta incluye aquellos basados en computador, entendiendo como tales aplicaciones informáticas que son capaces por sí solas de llevar a cabo actividades de manipulación del conocimiento. También detectan participantes híbridos (humano-computador), como aquellos en los que es necesaria la interacción para las actividades de gestión. Por su parte, los artefactos contienen representaciones de conocimiento utilizable, pero sin capacidad de manipular por sí mismo. A su vez, pueden ser incluidos en otros productos o recursos de información.

\section{«The purpose of an information inventory is to establish what information is held, or should be held, by an agency to sup- port their corporate activities and programs" (Office of Infor- mation Technology, 2002)}

El panorama se completa atendiendo a los recursos del medio ambiente de la organización, externos a la misma. Para la descripción de las fuentes de información se propone un conjunto de atributos que puedan ser aplicados a todos ellos. Si bien el marco general resulta interesante en cuanto a clasificación, en cambio los atributos propuestos son, a nuestro juicio, insuficientes para implementar un inventario de información.

Las propuestas existentes en la bibliografía no son homogéneas ni exhaustivas en lo que concierne a una herramienta fundamental como es el inventario de información. La variedad de enfoques y de contextos en los que realizar esta actividad ofrecen múltiples posibilidades. En cualquier caso, debe entenderse que los tipos de recursos a considerar pertenecerán a varios tipos de objetos diferentes, lo que obligará a trabajar con estructuras de datos y atributos diferentes, e independientes, para cada uno de ellos. Como mínimo, la estructura deberá permitir representar recursos de información, personas, flujos de información y procesos de actividad. Dado el desarrollo de los sistemas de bases de datos relacionales en la actualidad, y la progresiva integración de xml en los mismos, la solución más acertada pasa por el establecimiento de un esquema relacional, en una arquitectura abierta, que facilite la exportación de los datos y sus relaciones a xml, con la finalidad de integrarlo posteriormente con aplicaciones capaces de generar representaciones gráficas en el desarrollo de mapas de información.

\section{Bibliografía}

Applehans, W.; Globe, A.; Laugero, G. Managing knowledge. A practical web-based approach. Reading: Addison-Wesley, 1999.

Buchanan, S. J.; Gibb, F. The information audit: an integrated strategic approach. En: International journal of information management, 1998, v. 18 , n. 1 , pp. 29-47.

Bukowitz, W. R.; Williams, R. L. The knowledge management fieldbook. London: Financial Times/Prentice-Hall, 2000.

Burk, C. F.; Horton, F. W. InfoMap: a complete guide for discovering corporate information resources. Englewood Cliffs: Prentice-Hall, 1988.

David Skyrme Associates. Knowledge audit, 2002. Consultado en: 15-0403.

http://www.skyrme.com/services/kmaudit.htm

Di Mattia, S. S.; Blumenstein, L. "In search of the information audit: essential tool or cumbersome process?". En: Library journal, 2000, v. 125, n. 4 , pp. $48-50$.

Dobson, Chris. Beyond the information audit: checking the health of an organization's information system. Searcher, v. 10, n. 7, 2002. Consultado en: 06-04-03.

http://www.infotoday.com/searcher/jul02/dobson.htm

Henczel, S. "The information audit as a first step towards effective knowledge management: an opportunity for the special librarian". En: Inspel, 2000, v. 34, n. 3/4, pp. 210-226.

Henczel, S. The information audit. A practical guide. München: K. G. Saur, 2001.

Holsapple, C. W.; Joshi, K. D. "Organizational knowledge resources". En: Decision support systems, 2001, n. 31, pp. 39-54.

Hylton, A. Measuring \& assesing knowledge-value \& the pivotal role of the knowledge audit. London: Hylton Associates, 2002.

Nonaka, I.; Takeuchi, H. The knowledge creating company. New York: Oxford University Press, 1995.

Office of Information Technology, Department of Information Technology and Management. Information management. Inventory guideline. Sydney, 1997-2002.

Orna, E. Practical information policies. Aldershot: Gower, 1999.

Robertson, G. "Information auditing: the information professional as information accountant". En: Managing information, 1997, v. 4, n. 5, pp. 31-35.

St. Clair, G. "The information audit I: defining the process". En: InfoManage, 1997, v. 4, n. 6, pp. 5-6.

Stevens, L. Knowing what your company knows, 2000. Consultado en: 03-03-03.

http://www.destinationkm.com/print/default.asp? ArticleID $=613$

Tiwana, A. The knowledge management toolkit. Upper Saddle River, Prentice-Hall, 2002.

Jesús Tramullas, Departamento de Ciencias de la Documentación, Universidad de Zaragoza.

tramullas@unizar.es

http://tramullas.com 